\title{
Driver mutations as predictive biomarkers in lung cancer
}

\author{
Nicolas Girard
}

Published online: 7 January 2012

(C) Springer Science+Business Media, LLC 2012

\begin{abstract}
Treatment decisions for patients with lung cancer have historically been based upon tumor histology. Among the numerous molecular alterations observed in cancer cells, some specific mutations have been identified as being necessary and sufficient to drive tumor formation and maintenance. These "driver" mutations occur in genes that encode signaling proteins critical for cellular proliferation and survival. With the development of agents specifically designed to target these key proteins, the paradigm is changing to "personalized medicine," which consists of the individual genotyping of each patient tumor to identify driver mutations that are predictive biomarkers of the efficacy of these agents. This paper reviews the clinical and biologic evidence for the major driver mutations occurring in lung cancer.
\end{abstract}

Keywords Lung cancer - Chemotherapy - Targeted therapy · Anaplastic lymphoma kinase $\cdot$ Kinase $\cdot$ Driver mutation . EGFR $\cdot A L K \cdot H E R 2 \cdot B R A F \cdot K R A S \cdot P I K 3 C A \cdot A K T 1$. $M E K 1 \cdot M E T \cdot D D R 2 \cdot F G F R 1$

\footnotetext{
N. Girard

Department of Respiratory Medicine and Thoracic Oncology, Louis Pradel Hospital,

Hospices Civils de Lyon,

Lyon, France

N. Girard

UMR 754 Retrovirus and Compared Pathology,

Claude-Bernard University,

Lyon, France

N. Girard $(\bowtie)$

Hôpital Louis Pradel,

28, avenue Doyen Lépine,

69677 Lyon Cedex, France

e-mail: nicolas.girard@chu-lyon.fr
}

\section{Introduction}

Lung cancer is the most frequent cause of cancer-related death worldwide, accounting for more than 1 million deaths per year [1]. Lung cancer has been historically divided into two major histologic groups: small cell lung cancer (SCLC; $15 \%$ of cases) and non-small cell lung cancer (NSCLC; $85 \%$ of cases) [2]. NSCLC is composed of three major different types: adenocarcinoma, squamous cell carcinoma (SCC), and large cell carcinoma. Adenocarcinoma now accounts for more than $50 \%$ of all cases of lung cancer. Until recently, treatment of NSCLC was confined to surgery, radiotherapy, and chemotherapy. Optimization, including histology-driven decision-making of regimen [3], adjunction of the anti-angiogenic agent bevacizumab [4], and development of maintenance strategies [5], has marginally improved median survival of patients with metastastic tumor, ranging from 12 to 15 months in recent trials. Several molecular alterations have been reported to influence the prognosis of patients receiving chemotherapy, especially in the adjuvant setting [6]; however, these prognostic features are currently not included in the standard treatment strategy algorithm, in the absence of prospective validation. Overall, long-term survival remains lower than $15 \%$ in routine clinical care. Novel treatment strategies are needed.

Over the past decade, major progresses have been achieved in the understanding of the molecular bases of lung cancer. Data from genomic [7••], expression [8•], mutational $[9 \cdot \bullet, 10]$, and proteomic profiling studies [11] have been regarded for a long time by many clinicians as being obscure and out of touch with the overall poor reality of cancer care. Among the numerous molecular alterations observed in cancer cells, some specific mutations have been identified as being necessary and sufficient to drive tumor formation and maintenance (Fig. 1, Table 1) [12]. These 

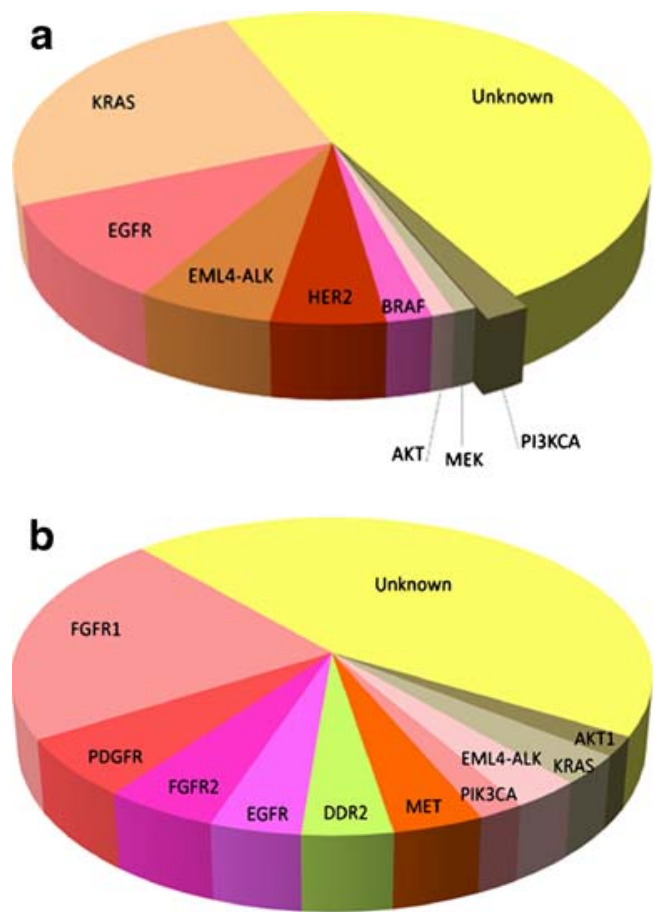

Fig. 1 Known driver mutations in lung adenocarcinoma a and squamous cell carcinoma $\mathbf{b}$. The mutations are mutually exclusive except for PIK3CA. (Data adapted from Ding et al. [9••], Hammerman et al. [10], and Pao et al. [12])

"driver" mutations occur in genes that encode signaling proteins critical for cellular proliferation and survival. Cancers may then rely upon the expression of these single mutant oncogenes for survival, even in the absence of other alterations, a concept referred to as "oncogene addiction" [13]. With the development of agents specifically designed to target these key proteins, the paradigm is changing from the traditional view of chemotherapy, which, even if binding specific proteins, aims at destroying cancer cells, to the potential use of targeted agents to specifically inhibit the causative oncogenic process by itself.

Over the past years, the systematic identification and targeting of driver mutations has become the leading challenge for the treatment of lung cancer, formalizing the concept of "personalized medicine," which consists of the individual genotyping of each patient tumor to identify driver mutations that are predictive biomarkers of the efficacy of specific targeted agents.

Epidermal growth factor receptor mutations

\section{Epidermal growth factor receptor activating mutations}

Epidermal growth factor (EGF) receptor (EGFR) mutations are the best illustration of the therapeutic relevance of identifying molecular clusters of NSCLC based on driver genetic alterations. Epidermal growth factor receptor is a transmembrane receptor harboring an intracytoplasmic tyrosine kinase domain that undergoes autophosphorylation after extracellular binding of a ligand, such as EGF, leading to homodimerization or hetero-dimerization with other HER family receptors [14••]. Following EGFR phosphorylation, key kinase-binding domains of downstream signaling proteins are activated, that control multiple cellular processes, including proliferation and survival [14••].

EGFR inhibitors have been developed along the clinical lines. The leading agents are orally available reversible, ATP-competitive inhibitors of the EGFR kinase: gefitinib (Iressa; AstraZeneca, Macclefields, UK) and erlotinib (Tarveva; Roche, Basel, Switzerland). Following promising phase 1 and 2 studies, gefitinib was compared to placebo in 1692 patients with refractory NSCLC [15]. A statistically significant difference in survival was not observed (5.6 vs 5.1 months, $P=0.11$ ).

At the same time clinical trials were ongoing, several groups took different approaches to identify predictors of response to EGFR tyrosine kinase inhibitors (TKIs). One approach hypothesized that patients who experienced striking responses to these agents had somatic mutations in EGFR that would indicate the essential role of the EGFR signaling pathway in the tumor [16]. Another approach involved high-throughput re-sequencing of exons encoding the kinase domains of several receptor tyrosine kinases, to

Table 1 Genes with driver mutations in non-small cell lung cancer: differences by histology

\begin{tabular}{lll}
\hline Mutation & Adenocarcinoma & Squamous cell carcinoma \\
\hline KRAS & $15 \%-25 \%$ & $<5 \%$ \\
EGFR* & $5 \%-15 \%$ & $5 \%-10 \%$ \\
$A L K$ & $5 \%-10 \%$ & $<5 \%$ \\
HER2 & $<5 \%$ & $0 \%$ \\
BRAF & $<5 \%$ & $0 \%$ \\
PIK3CA & $<5 \%$ & $<5 \%$ \\
$M E K 1$ & $<5 \%$ & $0 \%$ \\
MET & $<5 \%$ & $<5 \%$ \\
AKT1 & $0 \%$ & $<5 \%$ \\
$D D R 2$ & $0 \%$ & $4 \%$ \\
PDGFR & $0 \%$ & $5 \%-10 \%$ \\
$F G F R 2$ & $<5 \%$ & $5 \%-10 \%$ \\
SOX2** & $0 \%$ & $15 \%-25 \%$ \\
$F G F R 1^{* *}$ & $0 \%$ & $15 \%-25 \%$ \\
\hline
\end{tabular}

*EGFR kinase domain mutations are found predominantly in adenocarcinoma, while EGFR "vIII" mutations (that result from deletion of exons 2-7) are found predominantly in squamous cell carcinoma. Amplification may be identified in both histologies.

**Amplification. 
determine whether mutations in specific kinases played a causal role in NSCLC [17]. Investigators focused their analyses on patients with clinical characteristics predictive of response to EGFR inhibitors - a so-called "clinical enrichment" strategy [18, 19••]: Asians, never-smokers, women, adenocarcinoma tumors with bronchioloalveolar features. These studies demonstrated that somatic mutations in the EGFR kinase domain were present in a subset of NSCLCs, and were predictive of sensitivity to EGFR TKIs (Table 1, Fig. 1) $[16,17,20 \bullet]$. Most frequent EGFR mutations arise within the first 4 exons (exons 18 to 21 ) encoding the tyrosine kinase domain, and include in-frame deletions within exon 19 (codons 746-750; 44\% of cases), and L858R point mutations within exon 21 (41\% of cases).

These mutations confer ligand-independent activation and prolonged receptor kinase activity after ligand stimulation, and are sufficient for in vitro oncogenic transformation of fibroblasts and lung epithelial cells [20•, 21]. Additionally, tetracycline-regulatable mouse model systems indicate that expression of either EGFR exon 19 deletions or $L 858 R$ alleles in mouse lung epithelia leads to formation of tumors [22 $]$. These experiments demonstrate the driver nature of $E G F R$ mutations.

Back to the clinical side, four prospective randomized phase 3 trials are now available that all demonstrated that EGFR-activating mutations are the best predictor of efficacy of EGFR TKIs. These studies were conducted in patients with previously untreated metastatic EGFR-mutant NSCLC, and compared gefitinib or erlotinib to standard chemotherapy [23-26]. In these studies, contrary to trials conducted in unselected populations, response rates with EGFR TKIs ranged from $55 \%$ to $83 \%$. Median progression-free survival was significantly higher in the experimental arms (9.213.0 months) than in the chemotherapy arms (4.66.3 months). Median overall survival reached unprecedented values, higher than 27.0 to 30.5 months.

Given the major implications of these results for routine clinical practice, EGFR genotyping is now recommended as part of standard care to customize treatment with EGFR TKIs [27]. The Spanish group reported the feasibility of large-scale real-time screening of 2105 patients with lung cancer, 350 of whom had EGFR mutant tumors and 113 received treatment with erlotinib [28]. Median progressionfree and overall survival rates were strikingly similar to that reported in clinical trials, 14.0 and 27.0 months respectively.

\section{Acquired resistance to EGFR TKIs}

Besides EGFR non-activating mutations, such as exon 20 insertions and duplications, that are associated with limited response to EGFR TKIs [29], and molecular alterations, that may modulate the efficacy of EGFR TKIs, including PTEN loss, IGF-1R overexpression, and PIK3CA mutations (see below) [14••], acquired resistance to EGFR TKIs represents a major challenge in the management of EGFR-mutant lung cancer [30]. Analyses of tumor tissue from patients exhibiting tumor progression after initial response to these agents have revealed the presence of additional second-site $E G F R$ mutations, the most frequent being the EGFR T790M point mutation in exon 20 [31, 32], [33••]. The T790M mutation, while blocking the binding of erlotinib or gefitinib to the kinase ATP binding pocket, also causes drug resistance by increasing the affinity of the kinase domain for ATP [34]. The emergence of T790M mutation may involve a preexisting T790M clone, that becomes prominent after apoptosis of EGFR TKIs sensitive cells [35••]. Paradoxically, the T790M mutation may occur in vitro after repeated exposure of EGFR-mutant cells to EGFR TKIs [36].

Understanding the basis for acquired resistance has led to the identification of agents that may overcome acquired resistance. The presence of second-site mutations was thought to predict the efficacy of such "second-generation" EGFR inhibitors. Afatinib (BIBW2992; Boehringer-Ingelheim, Ingelheim am Rhein, Germany) is the "irreversible" EGFR TKI whose clinical development is the most advanced. Afatinib also targets HER2, and has demonstrated its potency to overcome T790M-mediated acquired resistance to erlotinib and gefitinib in vitro and in vivo [37]. However, the LUX-LUNG1 trial comparing afatinib to placebo in 585 patients with refractory NSCLC, without integrating EGFR genotyping in the inclusion criteria, did not reported statistically convincing results, even in the subgroup of tumors most likely to harbor EGFRactivating mutations [38]. Following promising in vivo results in mice, combination of afatinib with cetuximab was recently evaluated in a phase 2 trial, including 47 patients with EGFRmutant tumor, presenting with acquired resistance, 27 of which associated with T790M second-site mutations $[39 \bullet, 40]$. Disease control was observed in $92 \%$ of these patients, a promising figure for future phase 3 trials with this combination. Again, these data show the importance of using predictive driver mutations to select patients for specific targeted therapies.

Another mechanism of acquired resistance may involve a "kinase switch," the most studied example being MET amplification. MET is a receptor tyrosine kinase also known as the hepatocyte growth factor (HGF) receptor (HGFR) [41, 42]. The MET gene is located on chromosome $7 \mathrm{q} 21-\mathrm{q} 31$. Collectively, MET amplification has been found in about $20 \%$ of samples from patients with acquired resistance, with or without EGFR T790M mutations. As described below, MET activation may predict efficacy of MET inhibitors.

Fibroblast growth factor receptor-1 mutations

Similar to EGFR, fibroblast growth factor receptor-1 (FGFR1) is a transmembrane tyrosine kinase receptor, 
stimulating cell proliferation and survival. The $F G F R 1$ gene is located in $8 \mathrm{p} 12$ region. FGFR1 was recently demonstrated to harbor focal amplification in $15(9.7 \%)$ of 155 SCC tumors included in a large-scale single nucleotide polymorphism array genomic analysis [43••]. Using fluorescent in situ hybridization (FISH) with a more sensitive cutoff to define amplification, focal FGFR1 amplification was observed in $22 \%$ of cases in an independent cohort of 153 SCC tumors (Table 1, Fig. 1). Contrary to EGFR amplification, $F G F R 1$ amplification is exceptionally associated with activating FGFR1 mutations. FGFR1 amplification is exclusively observed in smokers and SCC tumors

Biologically, FGFR1 amplification leads to FGFR1 activation. In vitro studies demonstrated the driver nature of FGR1 amplification, which predicted the activity of PD173074 (TGen, Phoenix, AZ), a FGFR-specific TKI. Similar to EGFR T790M mutations, the ectopic expression of the FGFR1 V561M mutation, designed to block the FGFR1 ATP binding pocket, led to resistance to this drug. Taken together, these data suggest that FGFR1 amplification represents an exploitable target for SCC tumors, that predicts the efficacy of FGFR inhibitors. Such agents, including BIBF1120 (Boehringer-Ingelheim), are currently tested in phase $1 / 2$ trials.

\section{Anaplastic lymphoma kinase fusions}

The anaplastic lymphoma kinase (ALK) is a receptor tyrosine kinase, normally not expressed in the lung [44••]. Fusions of ALK with another upstream partner, the echinoderm microtubule-associated protein-like 4 (EML4), were recently found in NSCLC (Table 1, Fig. 1) [45••]. EML4$A L K$ fusions result from diverse small inversions within the short arm of chromosome $2[45 \bullet \bullet, 46,47 \bullet \cdot]$. At least nine different variants have been identified [47••]. Biologically, $E M L 4-A L K$ fusions result in protein oligomerization and constitutive activation of the kinase. In vitro, the fusions demonstrate gain-of-function properties [45••, 46]. Mice expressing an EML4-ALK variant under the control of a lung epithelial cell promoter develop multiple lung adenocarcinoma tumors [48].

The frequency of EML4-ALK translocation ranges from $3 \%$ to $7 \%$ in unselected NSCLC cohorts [45••, 46, 47••]. It has been associated with acinar, signet-ring variant, adenocarcinoma histology. The reference detection method is FISH, as reverse-transcriptase polymerase chain reaction (RT-PCR) and immunohistochemistry may lack sensitivity and specificity. Similar to EGFR mutations, EML4-ALK fusions are more frequently found in adenocarcinomas, in younger patients, and in never and light smokers [46, 47••]. $E M L 4-A L K$ translocations are usually found in tumors wild-

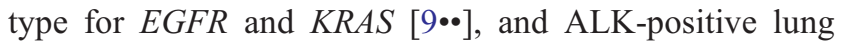
tumors are resistant to treatment with EGFR TKIs.
EML4-ALK fusions are strongly predictive of response to pharmacologic ALK inhibition. Crizotinib (Xalkori; Pfizer, New York, NY) is a small-molecule TKI with submicromolar activity against ALK and MET. Contrary to EGFR TKIs, the drug was directly evaluated in tumors harboring EML4-ALK fusion. After screening 1500 patients for the fusion, 82 patients were enrolled in the landmark phase 1 trial with crizotinib [49]. Overall, objective response and stable disease rates were $57 \%$ and $33 \%$, respectively. The estimated probability of 6 -month progression-free survival was $72 \%$. These results were recently confirmed in a phase 2 trial [50], and phase 3 trials are ongoing to compare crizotinib to standard chemotherapy in EML4-ALKrearranged tumors. Crizotinib is already approved in the US for $E M L 4-A L K$-rearranged tumors.

$E M L 4-A L K$ translocations are also predictors of the efficacy of heat shock protein 90 (HSP90) inhibitors [51]. EML4-ALK associates in complex with multiple cellular chaperones including HSP90. Inhibitors of HSP90 disrupt this complex, lead to degradation of EML4-ALK fusion protein, and produce tumor regression in vitro and in vivo. As for $E G F R$, acquired resistance to crizotinib has been associated with $A L K$ secondary mutations, involving both the "gatekeeper" residue (L1196M mutation) of the ATP binding pocket, and residues away from the kinase domain $[44 \bullet \cdot 51]$. Cell lines harboring these mutations remain equally sensitive to HSP90 inhibitors compared to ones without the secondary mutations.

\section{Discoidin domain receptor-2 mutations}

Discoidin domain receptor-2 is another transmembrane tyrosine kinase receptor, which binds collagen as its endogenous ligand, and is implicated in cell proliferation and migration. The $D D R 2$ gene is located in region $1 \mathrm{q} 23$ and was found to harbor activating mutations in a subset $(3.2 \%)$ of SCC lung tumors (Table 1, Fig. 1) [52••]. These mutations are not identified in other histologies, and are widely distributed on the gene, occurring both in the kinase domain and in other regions of the protein sequence. In vitro and xenograft studies demonstrated the oncogenic potency of the most frequent mutations (L239R and I638F), that were associated with gain-of-function of the receptor kinase activity and were transforming when introduced in fibroblast and epithelial cell lines.

$D D R 2$ mutations were found to be a strong predictor of efficacy for dasatinib (Sprycel; Bristol-Myers Squibb, New York, NY), a multikinase inhibitor with potent activity on SRC-family kinases, nonreceptor tyrosine kinases, and receptor tyrosine kinases (including Ephrin receptors, DDR1, and EGFR), both in vitro and in vivo in xenograft studies.

Clinically, one observation of a patient with SCC lung tumor harboring a $D D R 2$ S768R mutation was reported; 
partial response was observed after treatment with dasatinib, what further supports the use of these mutations as a predictive biomarker in this setting. A phase 2 trial with dasatinib is ongoing.

MET amplification and mutations

MET amplification, besides being found in tumors showing acquired resistance to EGFR TKIs, may be identified in EGFR TKI-naïve NSCLC tumors, at a frequency ranging from $1.4 \%$ to $21 \%$ (Table 1, Fig. 1) [53-55]. Such variability may be related to the method and cutoff used to define $M E T$ amplification. MET amplification occurs both in SCC and adenocarcinoma [54]. In adenocarcinoma, MET amplification, KRAS mutations, and EGFR amplification are independent events [53-55]. In vitro studies showed that MET amplification is associated with increased levels of phosphorylated MET. The ability of activated MET to initiate tumorigenesis has been shown in transgenic mouse models with ectopic expression of the activated receptor [56]. These data suggest that $M E T$ amplification is necessary and sufficient to drive the proliferation of cancer cells.

Mutations in the kinase domain of $M E T$ are rare in NSCLC. In a cohort of 188 lung adenocarcinomas, only three somatic $M E T$ mutations were identified, two in exon 13 encoding the juxtamembrane domain (R988del and Y1021N) and one in exon 18 encoding the kinase domain (G1260C) [9••]. The biological significance of these mutations is currently unknown. In addition, an intronic splice variant leading to exon 14 deletions has also been reported in about $2 \%-3 \%$ of NSCLC tumors in Japanese cohorts [53, 57]. Preliminary data indicate that this mutation leads to delayed receptor down-regulation and enhanced ligandmediated proliferation [57].

Specific small-molecule MET inhibitors are being developed [58]. METMAb, a monovalent monoclonal antibody that specifically binds the MET receptor, has been evaluated in combination with erlotinib versus erlotinib alone as second-line and third-line treatment in unselected patients with NSCLC [59•]. In this study, MET expression level at immunohistochemistry was a significant predictor of survival benefit.

\section{HER2 mutations}

Like EGFR, HER2 (ERBB2/human EGFR related (HER)-2) protein is a member of the HER family of receptor tyrosine kinases. HER2 has no identified ligand and forms homodimers or hetero-dimers with other members of the HER family [60]. The protein is overexpressed in about $20 \%$ of NSCLCs, but gene amplification occurs in only $2 \%$ of cases [61]. Early clinical trials with trastuzumab, a humanized anti-HER2 monoclonal antibody that is active in HER2- amplified breast cancer, have reported modest or disappointing effects in unselected NSCLC [62]. Interestingly, HER2 mutations are present in $2 \%$ of NSCLCs (Table 1, Fig. 1) [61]. The mutations involve in-frame insertions in exon 20, mostly in the amino acid sequence YVMA at codon 776 . Similar to EGFR mutations, HER2 mutations are more frequent in never smokers, females, Asian patients, and those with adenocarcinoma. HER2 mutations are not present in tumors harboring EGFR or KRAS mutations.

Biologically, HER2 insertions lead to constitutive activation of the receptor. Transgenic mice expressing the HER2 YVMA mutant develop lung adenosquamous carcinomas; in these models, significant tumor shrinkage was observed when combining afatinib with rapamycin, an inhibitor of the downstream effector mammalian target of rapamycin (mTOR) [63]. Consistent with this, a trial using afatinib has shown promising activity in patients with HER2 mutant lung adenocarcinoma [64].

Non-receptor kinase mutations

\section{KRAS mutations}

KRAS is a GTPase normally tethered at the cell membrane that is activated by growth factor transmembrane receptors, such as EGFR, HER2, ALK, MET, and FGFR. Point mutations involving amino acids 12,13 , and 61 lead to impaired GTPase activity, resulting in constitutive activation of downstream signaling cascades [65]. KRAS mutations are found in $20 \%-30 \%$ of lung adenocarcinoma tumors in Caucasian patients but are rarer in Asian populations (Table 1, Fig. 1) $[65,66 \bullet \bullet, 67]$. These mutations are mutually exclusive with other driver mutations, and are more frequent in smokers. Mice expressing an inducible mutant $K R A S$ in lung epithelial cells develop lung adenocarcinoma tumors that regress after silencing of the transgene [68・•].

Unfortunately, no effective targeted agents have yet been developed against mutant $K R A S$. An obstacle to the development of specific RAS inhibitors is that mutant $R A S$, which has gained constitutive activity, has lost its normal enzymatic function. Sorafenib (Nexavar; Bayer, Leverkusen, Germany) is a BRAF inhibitor, with multikinase inhibition potential against Raf1, BRAF, vascular endothelial growth factor (VEGF) receptor (VEGFR)-1, -2, and -3, platelet-derived growth factor receptor (PDGFR), CD117/KIT, and receptor tyrosine kinase (RET). Given the direct interaction of RAS with RAF, sorafenib has been evaluated, after failure of platinbased chemotherapy, in 57 patients with $K R A S$-mutant NSCLC [69]. Overall, disease control was observed in 53\% of patients after 6 weeks of treatment; progression-free survival was however limited (2.3 months). A more promising approach may involve combined PI3K and MEK inhibition, which both are downstream partners of RAS [69]. 


\section{PIK3CA mutations}

Phosphatidylinositol 3-kinases (PI3K) are lipid kinases that regenerate phosphatidylinositol-3-phosphate, a key mediator between growth factor receptors and intracellular downstream signaling pathways [71]. The main catalytic subunit of PI3K is the $\mathrm{p} 110 \alpha$ isoform which is encoded by the PIK3 catalytic subunit alpha (PIK3CA) gene. Mutations in PIK3CA occur in about $2 \%$ of cases (Table 1, Fig. 1) [72], affecting residues E542 and E545 in exon 9 encoding the catalytic domain [9••, 72, 73]. These mutations are as frequent in SCC as in adenocarcinoma, and, contrary to other driver mutations that are mutually exclusive, may occur in EGFR-mutant tumors [73]. Mutant PIK3CAs display gain of enzymatic function in vitro and may induce oncogenic cellular transformation [74]. GDC-0941 (Genentech, South San Francisco, CA) is a specific PIK3CA inhibitor that is being tested in a phase 2 trial following a favorable safety profile in phase 1 trials [75].

\section{AKT mutations}

Protein kinase B (AKT1) is a serine-threonine kinase that is activated by PIK3CA and mediates PI3K signaling [76]. A major recurrent $A K T 1$ mutation $(\mathrm{E} 17 \mathrm{~K})$ has been identified in several solid tumors, including breast, colon, and ovarian cancer [77]. Collectively, the incidence of AKT1 mutations in NSCLC is about $1 \%$ (Table 1, Fig. 1). These mutations have only been identified in SCC tumors and mostly in cohorts of Western patients [77].

The E17K change occurs in the AKT pleckstrin homology domain. This mutation alters the phosphoinositide-binding pocket, is transforming in vitro, and leads to PI3Kindependent AKT1 activation. The E17K mutation does not alter sensitivity to ATP-competitive AKT inhibitors. MK2206 (Merck, Whitehouse Station, NJ), an allosteric AKT inhibitor, is being tested in phase 1 trials.

\section{BRAF mutations}

BRAF (V-raf murine sarcoma viral oncogene homolog B1) is a serine/threonine kinase linking RAS GTPases to downstream proteins of the MAPK family that control cell proliferation [78]. In NSCLC, BRAF mutations are found in $1 \%$ $3 \%$ of tumors, mostly of adenocarcinoma subtype (Table 1 , Fig. 1) [9••, 79, 80]. Contrary to melanomas, NSCLC mostly harbor non-V600 mutations ( $88 \%$ of cases), including the L596V mutation in the kinase domain and the G468A in the $\mathrm{G}$ loop of the activation domain $[79,80]$. BRAF mutations are mutually exclusive with EGFR and $K R A S$ mutations. Biologically, $B R A F$ mutations display elevated kinase activity and lead to constitutive activation of extracellular signal-regulated kinase (ERK) 1/2.
Sorafenib has been evaluated in NSCLC mostly as an anti-angiogenic agent in combination with chemotherapy [81]. Other inhibitors are currently evaluated specifically in BRAF-mutant NSCLC, such as GSK2118436 (GlaxoSmithKline, London, UK).

\section{MEK mutations}

MEK1 is a serine-threonine kinase downstream of BRAF, that activates ERK1/2 [82]. In cancers, at least three mutations have been found in $M E K 1$, including Q56P, K57N, and D67N [83]. All occur in the non-kinase portion of the kinase. Somatic mutations in MEK1 have been identified in $1 \%$ of NSCLCs, predominantly adenocarcinomas (Table 1, Fig. 1) [83]. $M E K 1$ mutations are mutually exclusive with EGFR, KRAS, HER2, PIK3CA, and BRAF mutations. The $\mathrm{K} 57 \mathrm{~N}$ and Q56P mutants display gain-of-function properties in vitro [83], and cells harboring these mutations can display sensitivity to the small-molecule non-ATP competitive MEK inhibitor, AZD6244 (AstraZeneca). AZD6244 has been used to treat NSCLC, in a trial comparing the drug to pemetrexed as second-line treatment.

\section{Conclusions}

The identification of multiple clinically relevant "driver mutations" in NSCLC has led to an improved understanding of lung cancer pathogenesis. A major hope now is that knowledge of these lesions can be used to improve patient care, as these mutations are the best predictors of efficacy of specific targeted therapies. To this end, multiple academic centers have begun to develop multiplex mutational profiling assays in clinical molecular diagnostic facilities, in order to genotype prospectively patients' tumors [28••, 84••]. Such effort requires development of multiplex assays [85•], and high-level quality control to ensure consistency in testing methods [86].

Besides a static molecular picture of NSCLC cases at time of diagnosis, systematic re-biopsy of tumors harboring driver mutations and presenting with acquired resistance to specific inhibitors is emerging as a crucial step to identify targetable secondary mutations and possible phenotypic change. In a series of 37 patients with EGFR-mutant adenocarcinoma, presenting with acquired resistance to EGFR TKIs, five (14\%) tumors transformed into SCLC, and three $(8 \%)$ tumors into spindle-cell sarcomatoid tumors [33]. Repeated phenotypic and molecular assessment is then mandatory to guide treatment decisions throughout the course of the disease.

To conclude, driver mutations are predictive biomarkers of response to specific targeted therapies. While the identification of EGFR mutations was based on empiric clinical 
observation of response in a small number of patients in whom mutations were discovered, the current development of targeted agent is directly linked with the early identification of predictive biomarkers leading to select the accurate population to which the drugs is specifically delivered. From bench to bedside, personalized medicine represents a major revolution in the treatment of lung cancer.

Disclosure No potential conflicts of interest relevant to this article were reported.

\section{References}

1. American Cancer SocietyCancer Facts and Figures 2007, American Cancer Society, Atlanta, GA (2007)

2. World Health Organization, WHO histological classification of tumours of the lung, WB Travis, A Brambilla, HK MullerHermelinck, CC Harris, Editors, World Health Organization Classification of Tumours. Pathology and Genetics of Tumours of the Lung, Pleura, Thymus and Heart, IARC Press, Lyon, France (2004), p 10

3. Scagliotti GV, Parikh P, von Pawel J, et al. Phase III study comparing cisplatin plus gemcitabine with cisplatin plus pemetrexed in chemotherapy-naive patients with advanced-stage non-small-cell lung cancer. J Clin Oncol. 2008;26:3543-51.

4. Sandler A, Gray R, Perry MC, et al. Paclitaxel-carboplatin alone or with bevacizumab for non-small-cell lung cancer. N Engl J Med. 2006;355:2542-50.

5. Cappuzzo F, Ciuleanu T, Stelmakh L, et al. Erlotinib as maintenance treatment in advanced non-small-cell lung cancer: a multicentre, randomised, placebo-controlled phase 3 study. Lancet Oncol. 2010;11:521-9.

6. Olaussen KA, Dunant A, Fouret P, et al. DNA repair by ERCC1 in non-small-cell lung cancer and cisplatin-based adjuvant chemotherapy. N Engl J Med. 2006;355:983-91.

7. •- Weir BA, Woo MS, Getz G, et al. Characterizing the cancer genome in lung adenocarcinoma. Nature 2007; 450: 893-898. This paper reports on the results of systematic genomic profiling of a large cohort of adenocarcinoma tumors, providing researchers with landmark reference data.

8. • Motoi N, Szoke J, Riely GJ, et al. Lung adenocarcinoma: modification of the 2004 WHO mixed subtype to include the major histologic subtype suggests correlations between papillary and micropapillary adenocarcinoma subtypes, EGFR mutations and gene expression analysis. Am J Surg Pathol 2008; 32: 810-827. This paper reports on the results of expression profiling of a large cohort of adenocarcinoma tumors which were fully characterized pathologically

9. •- Ding L, Getz G, Wheeler DA, et al. Somatic mutations affect key pathways in lung adenocarcinoma. Nature 2008; 455: 10691075. This paper reports on the results of systematic screening for somatic mutations in oncogenes in a large cohort of adenocarcinoma tumors. Besides showing mutual exclusivity of well-known driver mutations, these data represent a basis for future research

10. Hammerman P, Sivachenko A, Pho A, et al. Genomic characterization and targeted therapeutics in squamous cell lung cancer. J Thorac Oncol 2011;6:S39 (abstract)

11. Rikova K, Guo A, Zeng Q, et al. Global survey of phosphotyrosine signaling identifies oncogenic kinases in lung cancer. Cell. 2007;131:1190-203.
12. Pao W, Girard N. New driver mutations in non-small-cell lung cancer. Lancet Oncol. 2011;12:175-80.

13. Gazdar AF, Shigematsu H, Herz J, et al. Mutations and addiction to EGFR: the Achilles 'heal' of lung cancers? Trends Mol Med. 2004; 10:481-6.

14. • Pao W, Chmielecki J. Rational, biologically based treatment of EGFR-mutant non-small-cell lung cancer. Nat Rev Cancer 2010;10:760-774. This is a landmark review about EGFR mutations biology in lung cancer.

15. Thatcher N, Chang A, Parikh P, et al. Gefitinib plus best supportive care in previously treated patients with refractory advanced nonsmall-cell lung cancer: results from a randomised, placebocontrolled, multicentre study (Iressa Survival Evaluation in Lung Cancer). Lancet. 2005;366:1527-37.

16. Lynch TJ, Bell DW, Sordella R, et al. Activating mutations in the epidermal growth factor receptor underlying responsiveness of non-small-cell lung cancer to gefitinib. N Engl J Med. 2004:350:2129-39.

17. Paez JG, Jänne PA, Lee JC, et al. EGFR mutations in lung cancer: correlation with clinical response to gefitinib therapy. Science. 2004;304:1497-500.

18. Girard N, Sima CS, Jackman DM, et al. Nomogram to predict the presence of EGFR activating mutation in lung adenocarcinoma. Eur Respir J 2011;doi:10.1183/09031936.00010111

19. •• Travis WD, Brambilla E, Noguchi M, et al. International association for the study of lung cancer/american thoracic society/ european respiratory society international multidisciplinary classification of lung adenocarcinoma. J Thorac Oncol 2011;6:244-285. This paper is a systematic review of pathological features of lung adenocarcinoma, with respect to clinical, radiological, and molecular aspects.

20. - Pao W, Miller V, Zakowski M, et al. EGF receptor gene mutations are common in lung cancers from "never smokers" and are associated with sensitivity of tumors to gefitinib and erlotinib. Proc Natl Acad Sci U S A 2004;101:13306-13311. This paper is the first report of EGFR mutations in lung cancer, integrating biologic characterization of mutations in vitro.

21. Greulich H, Chen TH, Feng W, et al. Oncogenic transformation by inhibitor-sensitive and -resistant EGFR mutants. PLoS Med. 2005;2:e313.

22. • Politi K, Zakowski MF, Fan PD, et al. Lung adenocarcinomas induced in mice by mutant EGF receptors found in human lung cancers respond to a tyrosine kinase inhibitor or to downregulation of the receptors. Genes Dev 2006;20:1496-1510. This paper describes the establishment of double-transgenic EGFR mutant mouse model.

23. Mitsudomi T, Morita S, Yatabe Y, et al. Gefitinib versus cisplatin plus docetaxel in patients with non-small-cell lung cancer harbouring mutations of the epidermal growth factor receptor (WJTOG3405): an open label, randomised phase 3 trial. Lancet Oncol. 2010;11:121-8.

24. Maemondo M, Inoue A, Kobayashi K, et al. Gefitinib or chemotherapy for non-small-cell lung cancer with mutated EGFR. N Engl J Med. 2010;362:2380-8.

25. Gervais R, Rosell R, Vergnenegre A, et al. The EURTAC (European Tarceva ${ }^{\circledR}$ vs Chemotherapy) study: interim results of a phase III randomized trial of erlotinib vs chemotherapy (CT) in advanced non-small-cell lung cancer (NSCLC) patients $(\mathrm{P})$ with epidermal growth factor receptor (EGFR) activating mutations. J Thorac Oncol 2011;6: S314 (abstract)

26. Zhou C, Wu YL, Chen G, et al. Efficacy results from the randomised phase III Optimal (CTONG 0802) study comparing first-line Erlotinib versus carboplatin (CBDCA) plus Gemcitabine (GEM), in Chinese advanced non small-cell lung cancer (NSCLC) patients (PTS) With EGFR activating mutations. J Thorac Oncol 2011;6: S315 (abstract) 
27. Azzoli CG, Baker Jr S, Temin S, et al. American Society of Clinical Oncology Clinical Practice Guideline update on chemotherapy for stage IV non-small-cell lung cancer. J Clin Oncol. 2009;27:6251-626.

28. • Rosell R, Moran T, Queralt C, et al. Screening for epidermal growth factor receptor mutations in lung cancer. N Engl J Med 2009;361:958-967. This paper reports on the first large-scale EGFR genotyping study.

29. Wu JY, Wu SG, Yang CH, et al. Lung cancer with epidermal growth factor receptor exon 20 mutations is associated with poor gefitinib treatment response. Clin Cancer Res. 2008;14:4877-82.

30. Jackman D, Pao W, Riely GJ, et al. Clinical definition of acquired resistance to epidermal growth factor receptor tyrosine kinase inhibitors in non-small-cell lung cancer. J Clin Oncol. 2010;28:357-60.

31. Pao W, Miller VA, Politi KA, et al. Acquired resistance of lung adenocarcinomas to gefitinib or erlotinib is associated with a second mutation in the EGFR kinase domain. PLoS Med. 2005;2:e73.

32. Kobayashi S, Boggon TJ, Dayaram T, et al. EGFR mutation and resistance of non-small-cell lung cancer to gefitinib. N Engl J Med. 2005;352:786-92.

33. • Sequist LV, Waltman BA, Dias-Santagata D, et al. Genotypic and histological evolution of lung cancers acquiring resistance to EGFR inhibitors. Sci Trans1 Med 2011;3:75ra26. This paper reports on a study evaluating phenotypic and molecular features of 37 EGFR-mutant lung adenocarcinomas showing acquired resistance to EGFR tyrosine kinase inhibitors. This paper reports the occurrence of phenotypic change from non-small cell lung cancer to small cell cancer in these tumors.

34. Yun CH, Mengwasser KE, Toms AV, et al. The T790M mutation in EGFR kinase causes drug resistance by increasing the affinity for ATP. Proc Natl Acad Sci U S A. 2008;105:2070-5.

35. •- Maheswaran S, Sequist LV, Nagrath S, et al. Detection of mutations in EGFR in circulating lung-cancer cells. $\mathrm{N}$ Engl $\mathrm{J}$ Med 2008;359:366-377. This paper is the first report of EGFR mutations identification in circulating tumor cells from non-small cell lung cancer, integrating biologic sequential characterization of mutations in vitro.

36. Ogino A, Kitao H, Hirano S, et al. Emergence of epidermal growth factor receptor T790M mutation during chronic exposure to gefitinib in a non small cell lung cancer cell line. Cancer Res. 2007;67:7807-14.

37. Li D, Ambrogio L, Shimamura T, et al. BIBW2992, an irreversible EGFR/HER2 inhibitor highly effective in preclinical lung cancer models. Oncogene. 2008;27:4702-11.

38. Miller VA, Hirsh V, Cadranel J, et al. Phase IIb/III double-blind randomized trial of afatinib (BIBW 2992, an irreversible inhibitor of EGFR/HER1 and HER2)+ best supportive care (BSC) versus placebo + BSC in patients with NSCLC failing 1-2 lines of chemotherapy and erlotinib or gefitinib (LUX-LUNG 1). EurJ Cancer 2010; suppl; LBA1 (abstract)

39. - Regales L, Gong Y, Shen R, et al. Dual targeting of EGFR can overcome a major drug resistance mutation in mouse models of EGFR mutant lung cancer. J Clin Invest 2009;119:3000-3010. This paper is the first to demonstrate the interest of dual targeting of EGFR to circumvent acquired resistance to EGFR inhibitors.

40. Janjigian YY, Groen HJ, Horn L, et al. Activity and tolerability of afatinib (BIBW 2992) and cetuximab in NSCLC patients with acquired resistance to erlotinib or gefitinib. J Clin Oncol 2011;29:7525 (abstract)

41. Engelman JA, Zejnullahu K, Mitsudomi T, et al. MET amplification leads to gefitinib resistance in lung cancer by activating ERBB3 signaling. Science. 2007;316:1039-43.

42. Bean J, Brennan C, Shih JY, et al. MET amplification occurs with or without T790M mutations in EGFR mutant lung tumors with acquired resistance to gefitinib or erlotinib. Proc Natl Acad Sci U S A. 2007;104:20932-10937.
43. •• Weiss J, Sos ML, Seidel D, et al. Frequent and focal FGFR1 amplification associates with therapeutically tractable FGFR1 dependency in squamous cell lung cancer. Sci Transl Med 2010 Dec 15;2:62ra93 This is the first report of FGFR1 amplification in squamous cell lung carcinoma.

44. •• Sasaki T, Jänne PA. New Strategies for Treatment of ALK Rearranged Non-Small Cell Lung Cancers. Clin Cancer Res 2011;doi:10.1158/1078-0432.CCR-11-1404 This is an extensive review of $A L K$ biology in lung cancer.

45. •- Soda M, Choi YL, Enomoto M, et al. Identification of the transforming EML4-ALK fusion gene in non-small-cell lung cancer. Nature 2007;448:561-566. This is the first study reporting on EML4ALK fusion in lung cancer.

46. Koivunen JP, Mermel C, Zejnullahu K, et al. EML4-ALK fusion gene and efficacy of an ALK kinase inhibitor in lung cancer. Clin Cancer Res. 2008;14:4275-83.

47. • Horn L, Pao W. EML4-ALK: honing in on a new target in nonsmall-cell lung cancer. J Clin Oncol 2009;27:4247-4253. This is an extensive review of the clinical features associated with ALKrearranged lung cancer.

48. Soda M, Takada S, Takeuchi K, et al. A mouse model for EML4-ALK-positive lung cancer. Proc Natl Acad Sci USA. 2008;105:19893-7.

49. Kwak EL, Bang YJ, Camidge DR, et al. Anaplastic Lymphoma Kinase Inhibition in Non-Small-Cell Lung Cancer. NEJM. 2010;363:1693-703.

50. Riely GJ, Kim DW, Crinò L, et al. Phase 2 data for crizotinib (PF02341066) in ALK-positive advanced non-small cell lung cancer (NSCLC): profile 1005. J Thorac Oncol 2011;6: S411 (abstract)

51. Katayama R, Khan TM, Benes C, et al. Therapeutic strategies to overcome crizotinib resistance in non-small cell lung cancers harboring the fusion oncogene EML4-ALK. Proc Natl Acad Sci U S A. 2011;108:7535-40.

52. •• Peter S. Hammerman PS, Sos ML, Ramos AH, et al.Mutations in the DDR2 Kinase Gene Identify a Novel Therapeutic Target in Squamous Cell Lung Cancer. Cancer Discovery 2011;1: doi:10.1158/2159-8274.CD-11-0005 This is the first report about DDR2 mutations in lung cancer.

53. Onozato R, Kosaka T, Kuwano H, Sekido Y, Yatabe Y, Mitsudomi T. Activation of MET by gene amplification or by splice mutations deleting the juxtamembrane domain in primary resected lung cancers. J Thorac Oncol. 2009;4:5-11.

54. Beau-Faller M, Ruppert AM, Voegeli AC, et al. MET gene copy number in non-small cell lung cancer: molecular analysis in a targeted tyrosine kinase inhibitor naïve cohort. J Thorac Oncol. 2008;3:331-9.

55. Cappuzzo F, Marchetti A, Skokan M, et al. Increased MET gene copy number negatively affects survival of surgically resected nonsmall-cell lung cancer patients. J Clin Oncol. 2009;27:1667-74.

56. Otsuka T, Takayama H, Sharp R, et al. c-Met autocrine activation induces development of malignant melanoma and acquisition of the metastatic phenotype. Cancer Res. 1998;58:5157-67.

57. Kong-Beltran M, Seshagiri S, Zha J, et al. Somatic mutations lead to an oncogenic deletion of met in lung cancer. Cancer Res. 2006;66:283-9.

58. Eder JP, Vande Woude GF, Boerner SA, LoRusso PM. Novel therapeutic inhibitors of the c-Met signaling pathway in cancer. Clin Cancer Res. 2009;15:2207-14.

59. - Spigel D, Ervin T, Ramlau R, et al. Final efficacy results from a randomized phase II study (OAM4558g) evaluating Met Mab or placebo in combination with erlotinib in advanced NSCLC. J Thorac Oncol 2011; 6: S359. This is the first study evaluating METMAb in lung cancer

60. Coussens L, Yang-Feng TL, Liao YC, et al. Tyrosine kinase receptor with extensive homology to EGF receptor shares chromosomal location with neu oncogene. Science. 1985;230:1132-9. 
61. Stephens P, Hunter C, Bignell G, et al. Lung cancer: intragenic ERBB2 kinase mutations in tumours. Nature. 2004;431:525-6.

62. Hirsch FR, Langer CJ. The role of HER2/neu expression and trastuzumab in non-small cell lung cancer. Semin Oncol. 2004;31:75-82.

63. Perera SA, Li D, Shimamura T, Raso MG, et al. HER2YVMA drives rapid development of adenosquamous lung tumors in mice that are sensitive to BIBW2992 and rapamycin combination therapy. Proc Natl Acad Sci U S A. 2009;106:474-9.

64. De Greve J, Teugels E, De Mey J, et al. Clinical activity of BIBW2992, an irreversible inhibitor of EGFR and HER2 in adenocarcinoma of the lung with mutations in the kinase domain of HER2neu. J Thorac Oncol 2009; 4:S307 (abstract)

65. Bos JL. Ras oncogenes in human cancer: a review. Cancer Res. 1989;49:4682-9.

66. •• Riely GJ, Kris MG, Rosenbaum D, et al. Frequency and distinctive spectrum of KRAS mutations in never smokers with lung adenocarcinoma. Clin Cancer Res 2008;14:5731-5734. This is a clinicalpathological study of all subtypes of KRAS mutations in lung cancer.

67. Jang TW, Oak CH, Chang HK, et al. EGFR and KRAS mutations in patients with adenocarcinoma of the lung. Korean J Intern Med. 2009;24:48-54.

68. $\bullet$ Fisher GH, Wellen SL, Klimstra D, et al. Induction and apoptotic regression of lung adenocarcinomas by regulation of a K-Ras transgene in the presence and absence of tumor suppressor genes. Genes Dev 2001;15: 3249-3262. This is a paper showing the development of KRAS-mutant lung adenocarcinoma in doubletransgenic mouse models.

69. Dingemans AM, Mellema WW, Groen HJM, et al. A Phase II study of sorafenib in patients with locally advanced and/or metastatic (stage IIIB or IV) non-small cell lung cancer (NSCLC) with a K-Ras mutation. EurJ Cancer 2011; suppl; LBA 27 (abstract)

70. Engelman JA, Chen L, Tan X, et al. Effective use of PI3K and MEK inhibitors to treat mutant Kras G12D and PIK3CA H1047R murine lung cancers. Nat Med. 2008;14:1351-6.

71. Jimenez C, Jones DR, Rodríguez-Viciana P, et al. Identification and characterization of a new oncogene derived from the regulatory subunit of phosphoinositide 3-kinase. EMBO J. 1998;17:743-53.

72. Samuels Y, Wang Z, Bardelli A, et al. High frequency of mutations of the PIK3CA gene in human cancers. Science. 2004;304:554.

73. Kawano O, Sasaki H, Endo K, et al. PIK3CA mutation status in Japanese lung cancer patients. Lung Cancer. 2006;54:209-15.

74. Ikenoue T, Kanai F, Hikiba Y, et al. Functional analysis of PIK3CA gene mutations in human colorectal cancer. Cancer Res. 2005;65:4562-7.

75. Sarker D, Kristeleit R, Mazina KET, et al. A phase I study evaluating the pharmacokinetics (PK) and pharmacodynamics (PD) of the oral pan-phosphoinositide-3 kinase (PI3K) inhibitor GDC0941. J Clin Oncol. 2009;273:3538.

76. Testa JR, Bellacosa A. AKT plays a central role in tumorigenesis. Proc Natl Acad Sci USA. 2001;98:10983-5.

77. Carpten JD, Faber AL, Horn C, et al. A transforming mutation in the pleckstrin homology domain of AKT1 in cancer. Nature. 2007;448:439-44.

78. Leicht DT, Balan V, Kaplun A, et al. Raf kinases: function, regulation and role in human cancer. Biochim Biophys Acta. 2007;1773:1196-212.

79. Davies H, Bignell GR, Cox C, et al. Mutations of the BRAF gene in human cancer. Nature. 2002;417:949-54.

80. Sasaki H, Kawano O, Endo K, et al. Uncommon V599E BRAF mutations in Japanese patients with lung cancer. J Surg Res. 2006;133:203-6.

81. Hanna NH, Pawel Jv, Reck M, Scagliotti G. Carboplatin/paclitaxel with/without sorafenib in chemonaive patients with stage IIIb-IV non-small cell lung cancer: interim analysis (IA) results from a randomized phase III trial (ESCAPE). J Thorac Oncol 2008; 3(S7): S268 (abstract 214)

82. Howe LR, Leevers SJ, Gómez N, et al. Activation of the MAP kinase pathway by the protein kinase RAF. Cell. 1992;71:335-42.

83. Marks JL, Gong Y, Chitale D, et al. Novel MEK1 mutation identified by mutational analysis of epidermal growth factor receptor signaling pathway genes in lung adenocarcinoma. Cancer Res. 2008;68:5524-8.

84. •• Johnson BE, Kris MG, Kwiatkowski D, et al. Clinical characteristics of planned 1000 patients with adenocarcinoma of lung (ACL) undergoing genomic characterization in the US: Lung Cancer Mutation Consortium (LCMC). J Thorac Oncol 2011; 6: S344 (abstract) This study shows the feasibility of driver mutations routine genotyping in academic centers.

85. - Pao W, Kris MG, Iafrate AJ, et al. Integration of molecular profiling into the lung cancer clinic. Clin Cancer Res 2009;15:5317-5322. This paper reports on the feasibility of multiplex genotyping to allow simultaneous identification of multiple driver mutations in lung adenocarcinoma.

86. Beau-Faller M, Degeorges A, Rolland E, et al. Crossvalidation study for epidermal growth factor receptor and KRAS mutation detection in 74 blinded non-small cell lung carcinoma samples: a total of 5550 exons sequenced by 15 molecular French laboratories evaluation of the EGFR mutation status for the administration of EGFR-TKIs in non-small cell lung carcinoma [ERMETIC] project-part 1. J Thorac Oncol. 2011;6:1006-15. 